\title{
Arterial Tortuosity Syndrome in a Neonate
}

Kyung Min Kim, Sun-Mi Hur, Ji Hong Yoon, Eun-Jung Lee, and Jae Young Lee

Department of Pediatrics, Seoul St. Mary's Hospital, College of Medicine, the Catholic University of Korea, Seoul, Korea

\section{ABSTRACT}

Arterial tortuosity syndrome (ATS) is a very rare autosomal recessive connective tissue disease characterized by generalized elongation and tortuosity of the medium- to large-sized arteries, and other systemic connective tissue manifestations. To date, this disease entity has not been reported in Korea. We report a case of ATS diagnosed in a neonate who presented with severe elongation and tortuosity of the aorta and its major branches, as well as the intracranial arteries. Additionally, the patient presented with a tortuous dilatation of the inferior vena cava, an aneurysmal dilatation of the extra-hepatic bile ducts, and an inguinal and sliding hiatal hernia. The diagnosis was confirmed using DNA sequencing analysis, and the patient demonstrated a compound heterozygosity for two novel mutations (c.738delG [p.Gln247Serfs*33] and c.362T>C [p.Ile121Thr]) in exon 2 of the SLC2A10 gene. Genetic analysis also confirmed that both parents were heterozygous carriers of the responsible mutations. Owing to such clinical manifestations, ATS is often misdiagnosed as other connective tissue diseases including Loeys-Dietz syndrome, Marfan syndrome, and Ehlers-Danlos syndrome. In patients presenting with a high index of suspicion, thorough clinical evaluation and screening for ATS including computed tomography or magnetic resonance angiography and target gene analysis are necessary for early diagnosis and management.

Key Words: Vascular malformation, Connective tissue, Arterial tortuosity, Aortic aneurysm, Joint laxity

\section{INTRODUCTION}

Arterial tortuosity syndrome (ATS) is a rare autosomal recessive connective tissue disease characterized by severe and widespread elongation and tortuosity of the aorta and mediumsized arteries associated with an increased risk of aneurysms and dissection, or stenosis ${ }^{1)}$. ATS patients present with characteristic facial features including an elongated face, downslanting palpebral fissures, a beaked nose, a high-arched palate, and micrognathia ${ }^{2}$. Other accompanying manifestations of connective tissue include hyperextensible skin, hernias, and skeletal abnormalities such as arachnodactyly, joint laxity, and pectus deformities ${ }^{2,3)}$. We report the first Korean case of ATS diagnosed in a neonate who presented with severe
Received: 22 September 2017

Revised: 18 October 2017

Accepted: 20 October 2017

Correspondence to: Jae Young Lee Department of Pediatrics, Seoul St. Mary's Hospital, 222 Banpo-daero, Seocho-gu, Seoul 06591, Korea

Tel: +82-2-2588-6189

Fax: +82-2-537-4544

E-mail: jaeyounglee@catholic.ac.kr

Copyright(c)

By Korean Society of Neonatology.

All right reserved.

This is an Open-Access article distributed under the terms of the Creative Commons Attribution Non-Commercial License (http://creativecommons.org/licenses/ by-nc/4.0), which permits unrestricted non-commercial use, distribution, and reproduction in any medium, provided the original work is properly cited. 
elongation and tortuosity of the major arteries, a dilated inferior vena cava, an aneurysmal dilatation of the extra-hepatic bile ducts, and an inguinal and siding hiatal hernia.

\section{CASE REPORT}

A male neonate was admitted for evaluation of a diaphragmatic hernia and cardiomegaly found on prenatal screening. He was born via a full-term uncomplicated vaginal delivery to healthy, nonconsanguineous parents without any known family history of genetic disease. His birth weight was $2,950 \mathrm{~g}\left(10-25^{\text {th }}\right.$ percentile), head circumference was $33.5 \mathrm{~cm}$ (10-25 ${ }^{\text {th }}$ percentile), and height was $51 \mathrm{~cm}\left(50-75^{\text {th }}\right.$ percentile). He was observed to have a slightly elongated face, micrognathia, and downslanting palpebral fissures. On physical examination, shallow respirations with decreased breathing sounds were noted. Heart sounds were regular without any specific murmurs. His abdomen looked slender. There was no significant difference in the blood pressure measured between the upper and lower extremities. Peripheral blood analysis results were within reference range. A chest radiograph revealed a gaseous shadow in the mid chest region just above the diaphragm, indicating a sliding hiatal hernia (Figure 1). Echocardiography showed no intracardiac abnormalities with a left aortic arch, but the thoracic descending

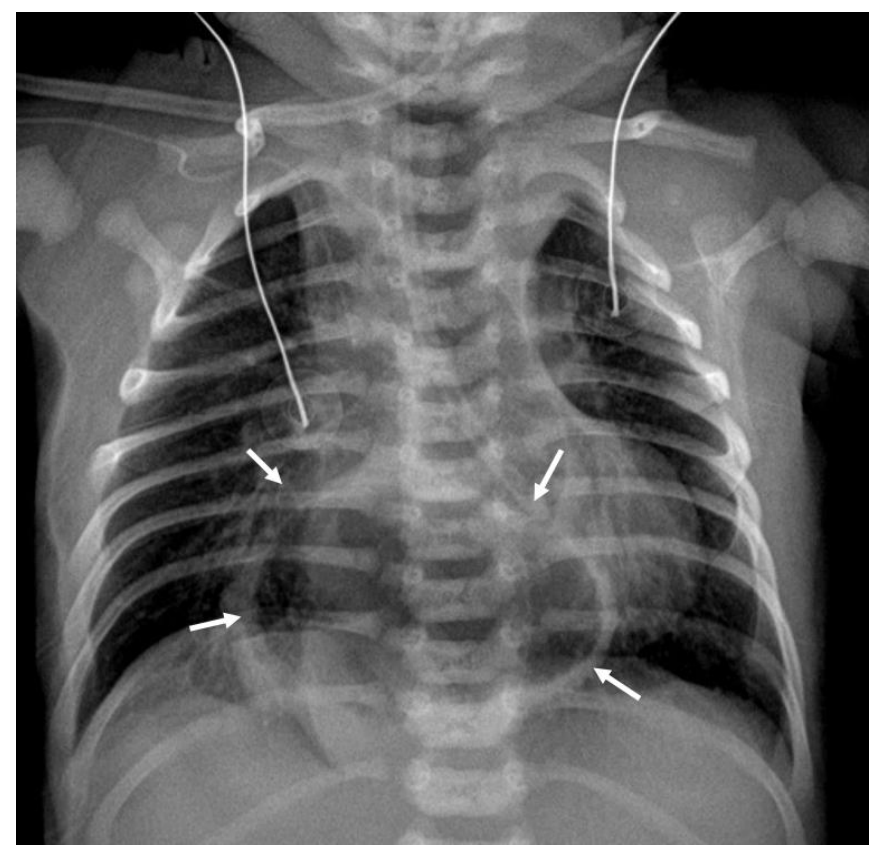

Figure 1. A gaseous shadow (arrows) indicating a sliding hiatal hernia. aorta could not be well visualized. Three-dimensional computed tomography angiography revealed a severely elongated and tortuous aorta and its main branches including the brachiocephalic artery, as well as the bilateral carotid, subclavian and iliofemoral arteries (Figure 2A). Additionally, a relative narrowing at the origin and early division of the main pulmonary artery, leading to an "inverted V" configuration of the pulmonary bifurcation was noted on axial imaging. The inferior vena cava was elongated, tortuous, and dilated (Figure 2B). Brain magnetic resonance angiography revealed severely tortuous cervical and intracranial arteries (Figure 3). A dilated fusiform extrahepatic bile duct was noticed on ultrasonographic examination of the liver (Figure 4). His hiatal hernia was successfully repaired and an inguinal hernia that was noted to have developed during followup was treated surgically. DNA sequencing genetic analysis subsequently confirmed the diagnosis of ATS. The patient demonstrated a compound heterozygosity for two mutations not reported previously (c.738delG [p.Gln247Serfs*33] and c.362T>C [p.Ile121Thr]) in exon 2 of the SLC2A10 gene. Genetic analysis performed in his parents revealed heterozygous mutations: c.738delG (p.Gln247Serfs*33) in his father and c.362T>C (p.Ile121Thr) in his mother. He is presently 11 months of age with normal somatic growth (weight at the $50-75^{\text {th }}$ percentile, height

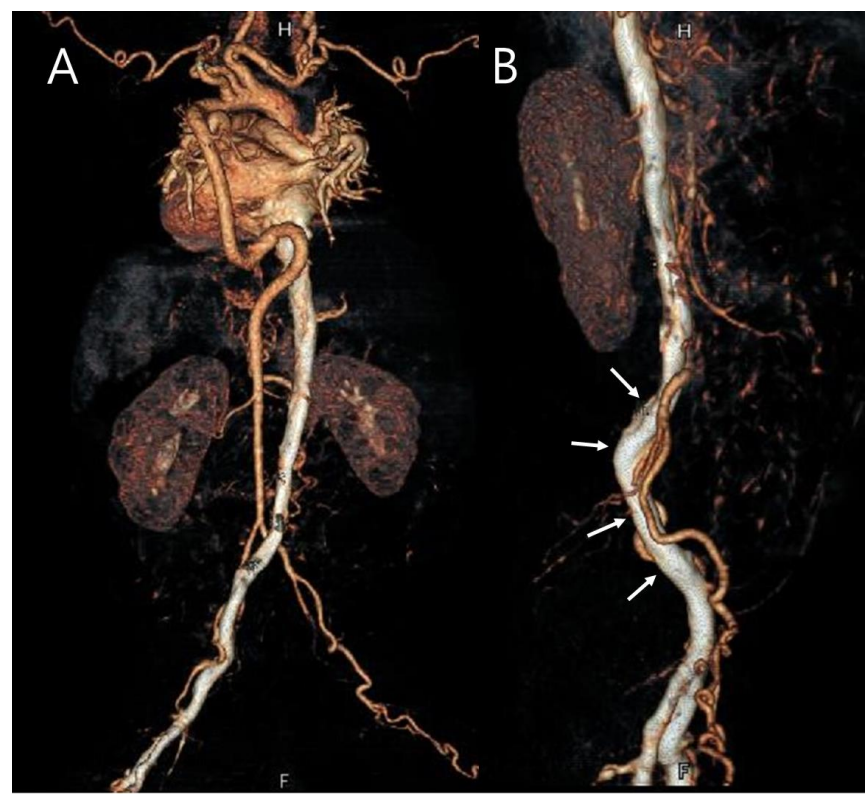

Figure 2. Three-dimensional computed tomography angiography shows severely elongated and tortuous aorta and its main branches including brachiocephalic artery, both carotid arteries, both subclavian arteries and both iliac-femoral arteries (A), and tortuous and dilated inferior vena cava (arrows)(B). 


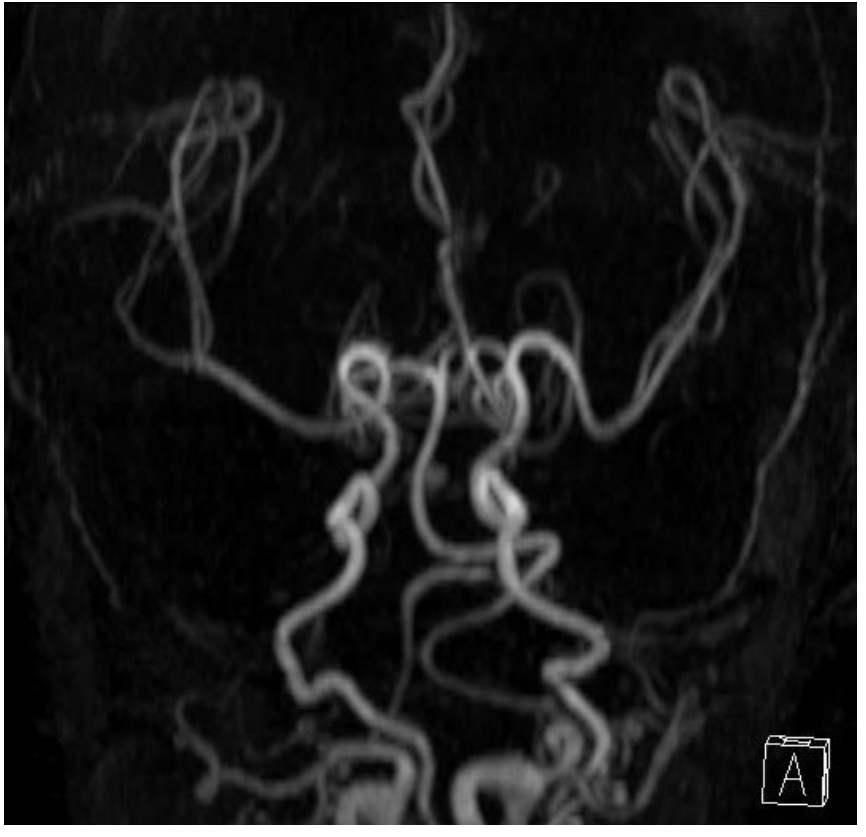

Figure 3. Brain magnetic resonance angiography shows severely tortuous cervical and intracranial arteries.

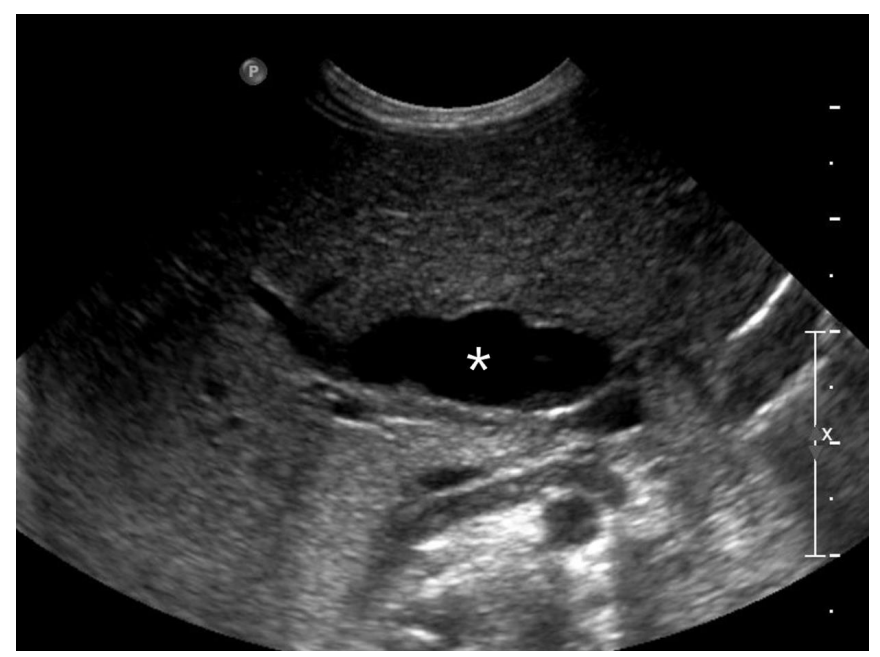

Figure 4. A dilated fusiform extrahepatic bile duct (*).

at the $50-75^{\text {th }}$ percentile, and head circumference at the $50-75^{\text {th }}$ percentile) and development. To date, there have been no reported complaints pertaining to the central nervous system or cardiovascular complications, and he is being closely monitored for disease progression.

\section{DISCUSSION}

We describe a case of ATS in a neonate who presented with se- vere and widespread involvement of his major arteries including the aorta and its major branches, cerebral and pulmonary arteries, as well as the inferior vena cava. Moreover, the patient also presented with an aneurysmal dilatation of the extra-hepatic bile duct, and inguinal and sliding hiatal hernias. This is the first case of ATS observed in Korea.

The clinical severity of ATS is variable. Although a few patients present with limited symptoms, the overall prognosis is very poor with reported mortality rates of approximately $41 \%$ before the age of 5 years ${ }^{4}$. Cardiovascular involvement remains the primary cause of mortality and morbidities in these patients. Regardless of age, this condition is associated with an increased risk of aneurysm formation and dissection of the aorta, in addition to the development of severe systemic or pulmonary hypertension and ischemic vascular events such as non-hemorrhagic stroke and infarction of abdominal organs secondary to arterial stenosis ${ }^{4-8)}$. Owing to such overlapping clinical manifestations, ATS is often misdiagnosed as other connective tissue diseases including Loeys-Dietz syndrome, Marfan syndrome, and Ehlers-Danlos syndrome. Initially, Loeys-Dietz syndrome was suspected in our patient, but this condition was excluded after gene analysis for the disease.

Clinical manifestations in our patient were similar to those previously reported in ATS patients in terms of generalized elongation and tortuosity of large- to medium-sized arteries and facial features. The initial clinical manifestations needing management were a sliding hiatal hernia and an inguinal hernia that was observed to have subsequently developed during follow-up. Skeletal manifestations were not evident because of the early age of the patient at the time of diagnosis. Involvement of the inferior vena cava and the extrahepatic bile ducts were unusual manifestations observed in our patient. To date, involvement of the venous system (innominate vein tortuosity) has been reported in only one case, and bile duct involvement has not been reported previously ${ }^{5}$. The findings observed in our patient suggest that compromised tissue integrity is not confined to blood vessels, but can occur even in the 'duct system' of the body.

The pathophysiological features of ATS include compromised vascular integrity in the arterial vascular beds and disorganized connective tissues overlying blood vessels and even non-vascular structures throughout the body leading to increased flexibility and reduced strength of the vessel wall and other connective tissues ${ }^{9}$. The gene causing ATS is SLC2A10, encoding glucose transporter type 10 (GLUT10), located at chromosome 
$20 \mathrm{q} 13^{9)}$. Recessive loss-of-function mutations of the SLC2A10 gene lead to disinhibition of the transforming growth factor beta signaling pathway and inhibition of proper extracellular matrix formation causing compromised vascular integrity and disorganized connective tissues ${ }^{2,9)}$. Expression of the SLC2A10 gene has been identified in several tissues, and high levels of SLC2A10 gene expression have been noted in the aortic vascular smooth muscle cells ${ }^{9,10)}$. In this study, DNA sequencing genetic analysis performed in the patient and his parents confirmed that the patient demonstrated a compound heterozygosity for two novel mutations not previously reported, and his parents were heterozygous carriers of the responsible mutations: c.738delG (p.Gln247Serfs*33) in his father and c.362T>C (p.Ile121Thr) in his mother. This finding confirmed the autosomal recessive inheritance pattern of ATS.

In conclusion, ATS should be considered among the differential diagnoses in patients who present with multiple hernias, manifestations of connective tissue disease, and vascular abnormalities in early infancy. In patients presenting with a high index of suspicion, a thorough clinical examination is necessary in addition to screening for ATS using computed tomography angiography, magnetic resonance angiography, and ultrasonography for examination of the abdominal organs. Moreover, target gene analysis is also essential for early diagnosis and timely management of this fatal disease

\section{CONFLICT OF INTEREST}

No potential conflict of interest relevant to this article was reported.

\section{REFERENCES}

1) Ertugrul A. Diffuse tortuosity and lengthening of the arteries. Circulation 1967;36:400-7.

2) Callewaert BL, Willaert A, Kerstjens-Frederikse WS, De Backer J, Devriendt K, Albrecht B, et al. Arterial tortuosity syndrome: clinical and molecular findings in 12 newly identified families. Hum Mutat 2008;29:150-8.

3) Franceschini P, Guala A, Licata D, Di Cara G, Franceschini D. Arterial tortuosity syndrome. Am J Med Genet 2000;91:141-3.

4) Wessels MW, Catsman-Berrevoets CE, Mancini GM, Breuning MH, Hoogeboom JJ, Stroink H, et al. Three new families with arterial tortuosity syndrome. Am J Med Genet A 2004;131:13443.

5) Moceri P, Albuisson J, Saint-Faust M, Casagrande F, Giuliano F, Devos C, et al. Arterial tortuosity syndrome: early diagnosis and association with venous tortuosity. J Am Coll Cardiol 2013; 61:783.

6) Kalfa D, Gronier C, Ly M, Le Bret E, Roussin R, Belli E. Giant aortic aneurysm in an infant with arterial tortuosity syndrome. Ann Thorac Surg 2012;94:e51.

7) Al-Khaldi A, Mohammed Y, Tamimi O, Alharbi A. Early outcomes of total pulmonary arterial reconstruction in patients with arterial tortuosity syndrome. Ann Thorac Surg 2011;92:698704.

8) Satish G, Nampoothiri S, Kappanayil M. Images in cardiovascular medicine. Arterial tortuosity syndrome: phenotypic features and cardiovascular manifestations. Circulation 2008; 117:e477-8.

9) Wetzel-Strong SE, Detter MR, Marchuk DA. The pathobiology of vascular malformations: insights from human and model organism genetics. J Pathol 2017;241:281-93.

10) Lee YC, Huang HY, Chang CJ, Cheng $\mathrm{CH}$, Chen YT. Mitochondrial GLUT10 facilitates dehydroascorbic acid import and protects cells against oxidative stress: mechanistic insight into arterial tortuosity syndrome. Hum Mol Genet 2010;19:3721-33. 